\title{
Preparation and Studying of Zeolite with Catalytic Properties From Silica and Bauxite Ores Local
}

\author{
Ragheed Yousif Ghazal $^{* 1}$, Tamarah Abdulsalam Younus ${ }^{2}$ \\ ${ }^{1,2}$ Department of Chemistry, College of Education for pure Science, University of Mosul, Mosul, Iraq \\ E-mail: ${ }^{1}$ ragheedghazal76@uomosul.edu.iq, ${ }^{2}$ tamarasalam17@gmail.com
}

(Received August 12, 2020; Accepted September 07, 2020; Available online March 01, 2021)

DOI: 10.33899/edusj.2020.127990.1104, (C) 2020, College of Education for Pure Science, University of Mosul.

This is an open access article under the CC BY 4.0 license (http://creativecommons.org/licenses/by/4.0/).

\begin{abstract}
The research included a method for preparing a zeolite by utilizing the components of clay mineral ores (silica and alumina). The two ores (/Local Silica and Bauxite) were studied through chemical analysis, X-ray diffraction and fluorescence. The results showed that they contained good quantities of these two substances, which were converted into sodium silicate and sodium aluminate, then the zeolite was prepared by hydrothermal method at $(\mathrm{pH}=11)$ in the presence of the structural directing agent (tri ethyl amine) then converted to the formula (H-zeolite) to improve its acidic properties, And then a number of measurements have been done for the prepared zeolite, which is a thermo gravimetric analysis (TGA), it showed that it contains amounts of moisture and crystallization water as well as its thermal stability at $\left(700^{\circ} \mathrm{C}\right)$. As for the results of X-ray diffraction (XRD), it showed its good crystallization rate and its possession of a crystalline cube system, and the measurement of Xray fluorescence (XRF) showed that it had a high percentage of silicon and aluminum, this indicates a balanced and equal interaction between these two materials when preparing of zeolite. The measurement of the scanning electron microscope (SEM) showed the high pore system and the fine particles that were around $(18.17 \mathrm{~nm})$, Finally the measurement of adsorption by the (BET) technique that explained the prepared zeolite have a high surface area $\left(244.3766 \mathrm{~m}^{2} / \mathrm{g}\right)$.
\end{abstract}

Keywords: zeolite, local silica and bauxite ores, hydrothermal synthesis, catalysts properties.

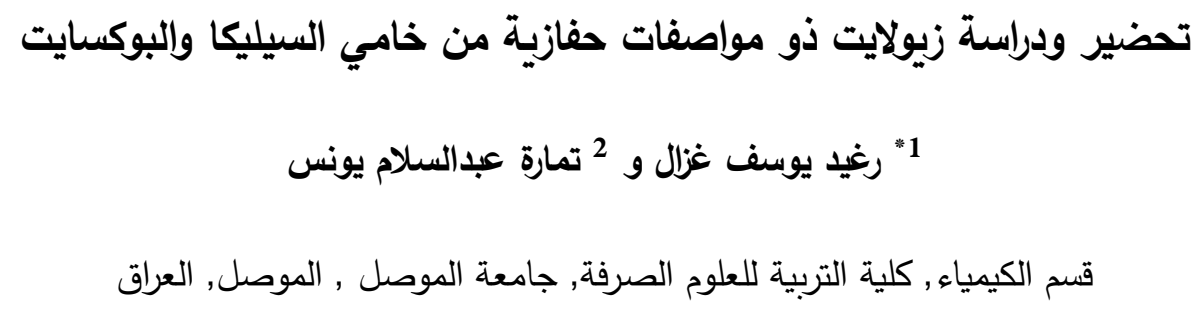

الخلاصة:

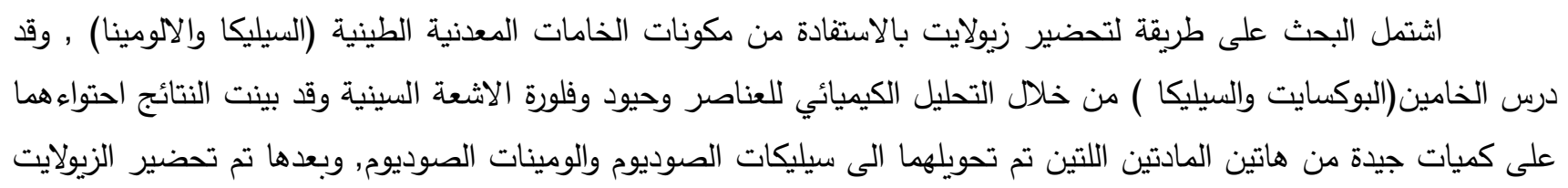


بالطريقة الهيدروحرارية عند( pH=11 ) وبوجود العامل الموجه للبنية (ثلاثي اثيل امين) ثم حول الى الصيغة (H-zeolite) لتحسين

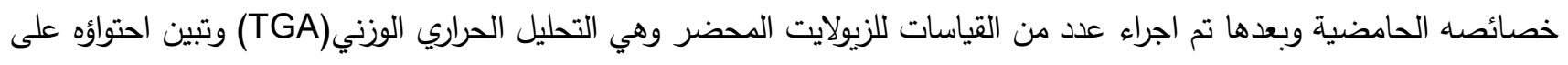

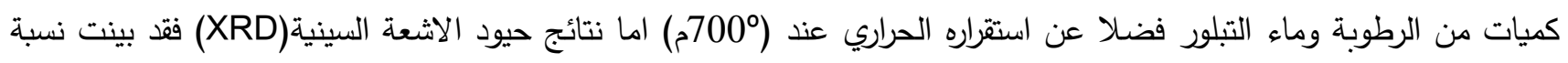
تبلوره الجيدة وامتلاكه نظاماً بلورياً مكعبياً, وبين قياس فلورة الاشعة السينية(XRF) احتواؤه على نسبة عالية من السيليكون والالمنيوم

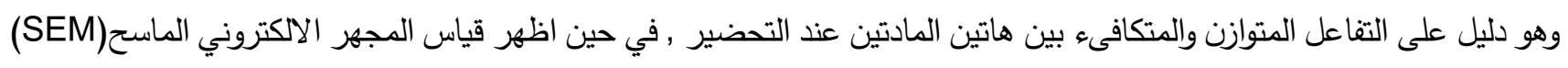

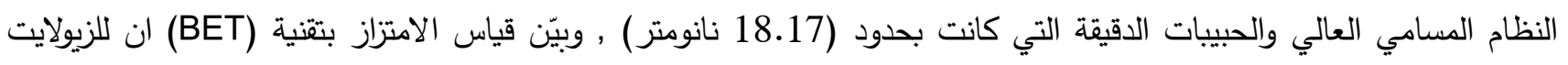

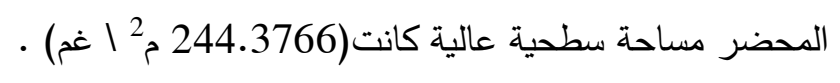

الكلمات المفتاحية : الزيولايت , خام السيليكا والبوكسايت , الطريقة الهيدروحرارية , الخصائص الحفازية.

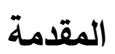

ان الزيولايتات عبارة عن سيليكات الألمنيوم المائية المتبلورة مع احتوائه على بعض الأيونات الموجبة مثل الصوديوم

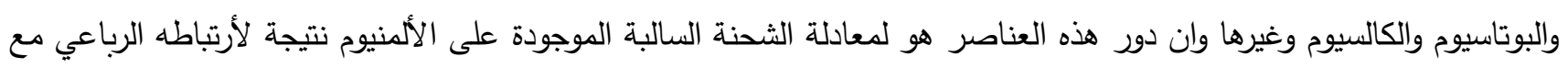

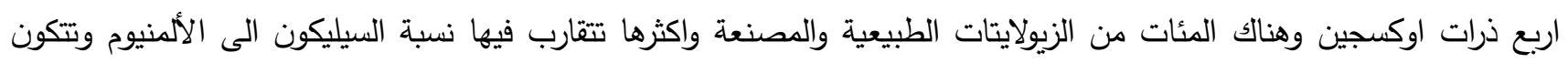

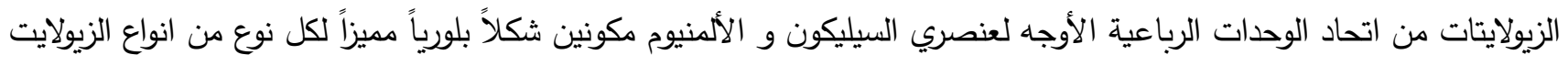

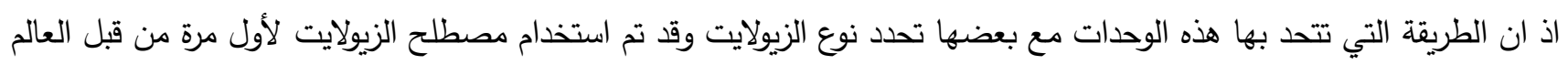

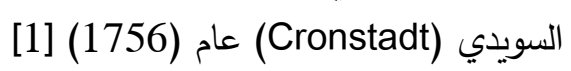

ويوصف الزيولايت بان له تراكيب مسامية ناتجة من طريقة ارتباط الوحدة المسـماة بـ (Cage (م-Cage ) وترتبط مع بعضها محددةً تركيب الزيولايت البلوري من خلال العوامل الموجهة للبنية وهي مركبات عضوية مثل مثل بروميد رباعي بروبيل الأمونيوم (TPABr)

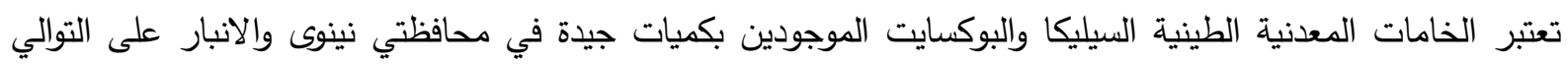

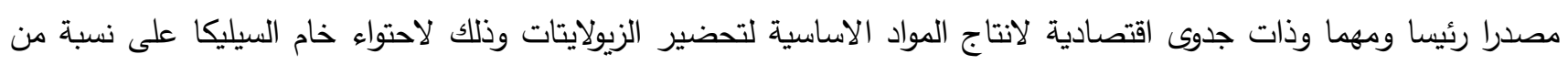

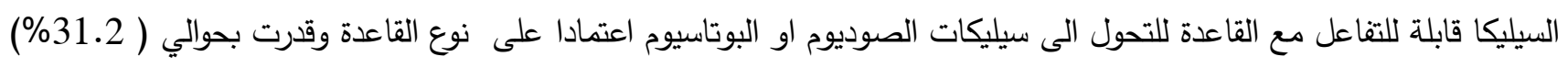
,اما خام البوكسايت فيحتوي على نسبة (54.02\%) من الالمنيوم قابلة للتحول الى الومينات الصوديوم , وتعد هاتين المادتين

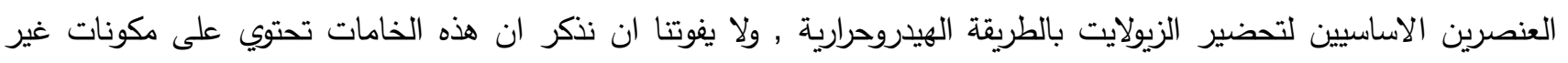
مرغوبة مثل الحديد والكاربونات فضلا عن الجبسوم في خام السيليكا [3]. وعند تحضير الزيولايت فان هناك عدد من العوامل والظروف التي تؤثر بشكل رئيسي على التركيب البلوري و حجم المسامات

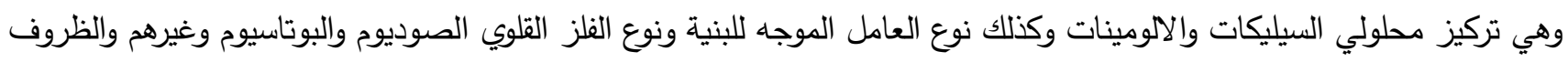
الاخرى من درجة الحرارة والضغط وزمني التفاعل والتبلور [2].

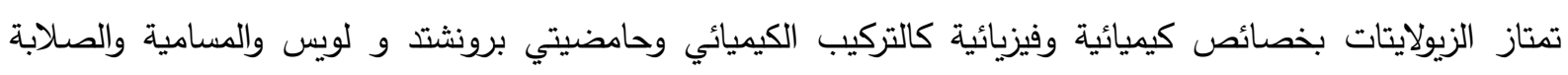

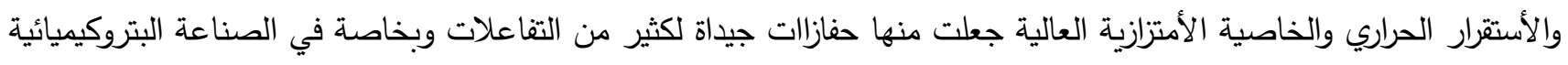

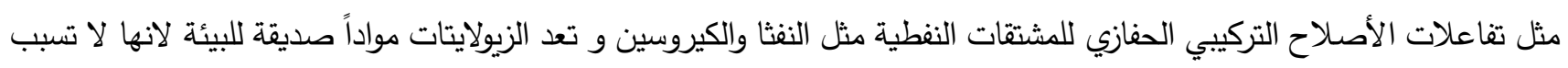
التآكل مثل الأحماض المعدنية (الكبريتيك والهيدروكلوريك وغيرها) وكذلك سهولة استعادة فعاليتها بطرق غير معقدة وذات تكلفة اقل [4] 


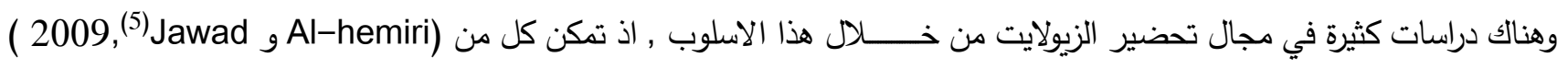

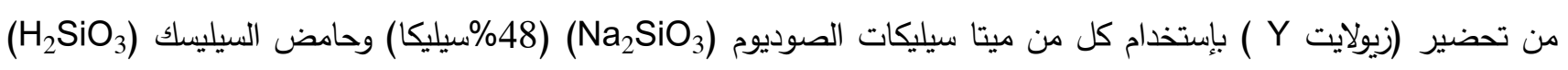

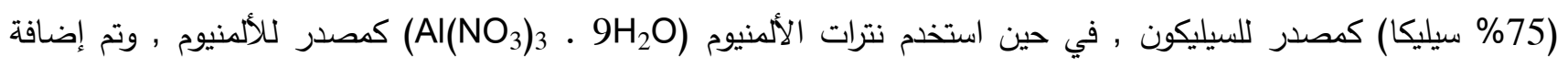

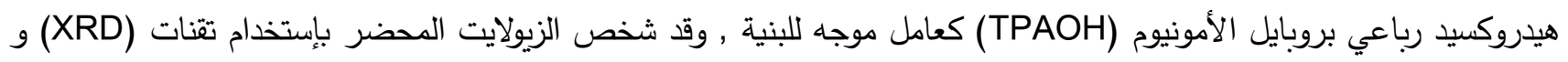

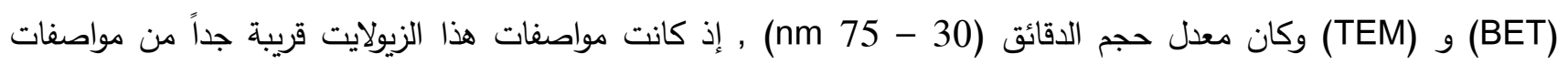

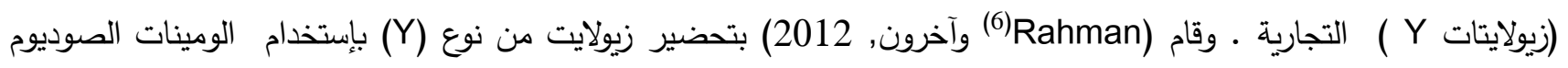

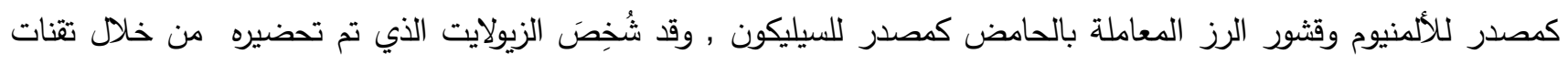
(BRD) و (XET) وكان معدل حجم المسامات لهذا الزيولايت(SE 21.22) وقد استخدم لتنقية المياه اذ تبين فعاليته العالية

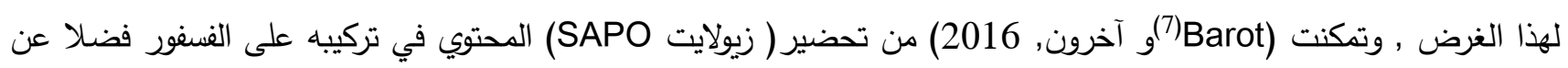

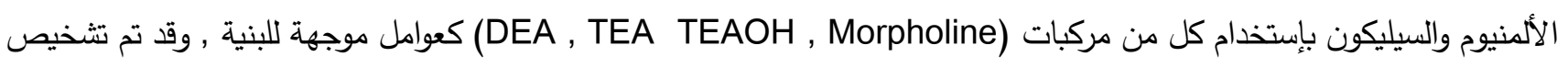

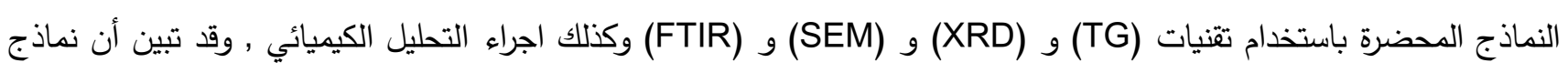

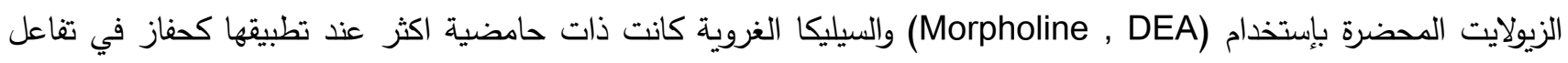

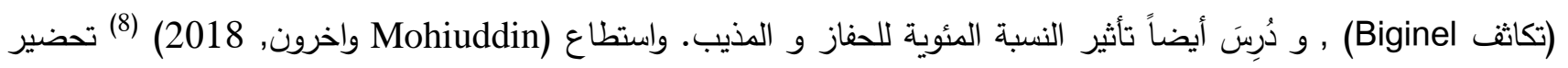

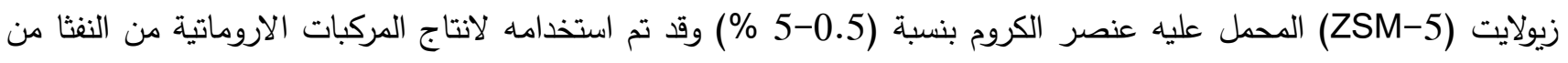

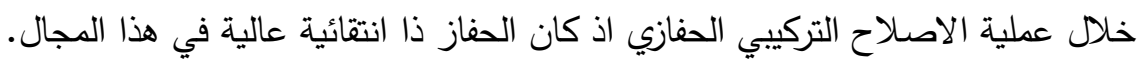

\section{الجزء العملي}

1-اختيار ودراسة الخامات الطبيعية قيد البحث:

أختير نموذج خام السيليكا من منطقة الحمدانية في محافظة نينوى بعد ان تم تحليلها والتعرف على محتوياتها اذ بينت التحاليل احتواءها على كميات جيدة من السيليكا , كما استخدم خام البوكسايت باعتباره مصدر مهم للالمنيوم , وقد تم الحصول علئيه

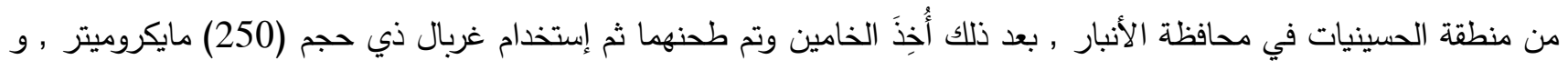

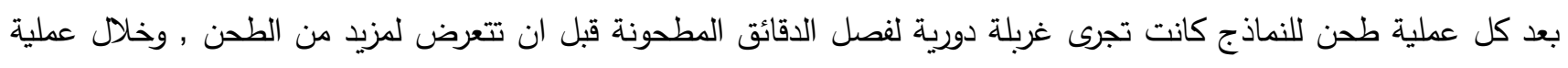

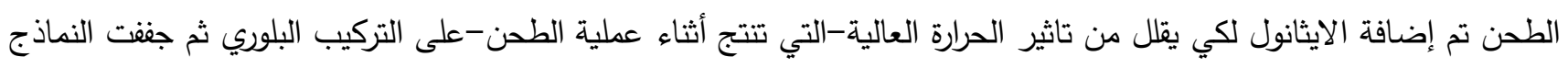
بدرجة حرارة (110)ْ م لمدة (6-8) ساعات وتم حفظهما في قناني محكمة الإغلاق (9).

\section{2- تحليل الخامات المعدنية الطينية:}

1-2

اذ اجري التحليل الكيميائي لخام السيليكا في مختبرات معمل سمنت الحدباء في ناحية حمام العليل , والتي اشتملت على

تقدير كل من السيليكون والامنيوم والحديد والكالسيوم والمغنيسيوم و الكبريتات, وتم إجراء التحليل الكيميائي لخام البوكسايت في دراسة سابقة(10) وقد شملت قياس نسب كل من الالمنيوم و السيليكون والمغنيسيوم والحديد والكالسيوم والصوديوم .

2-2 قياس حيود الاشعة السينية(XRD):

تم قياس حيود الأشعة السينية لخام السيليكا بجهاز من نوع Xpert Phillips Holland باما بالنسبة لخام البوكسايت فقد

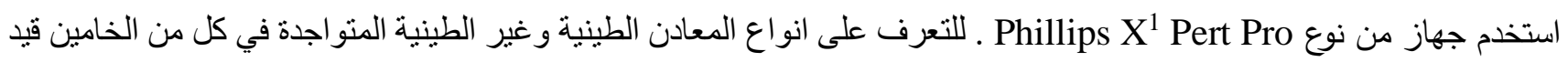


(XRF) قاس قلورة الاشعة السينية) اجريت قياسات فلورة الأشعة السينية لخامي البوكسايت والسيليكا بإستخدام جهاز من نوع 2000 G.N.R. TX للتعرف على نسبة العناصر (الألمنيوم والسيليكون والحديد والكالسيوم والمغنيسيوم ) ومقارنتها مع نتائج التحليل الكيميائي.

3- تحضير الزيولايت

3-3 الحصول على سيليكات الصوديوم من خامي السيليكا والبوكسايت :

يوضع (100 غم) من النموذج في دورق دائري سعة (500) مل ويضاف إليه (250) مل من محلول (0.5) مولاري ( ثمaOH)

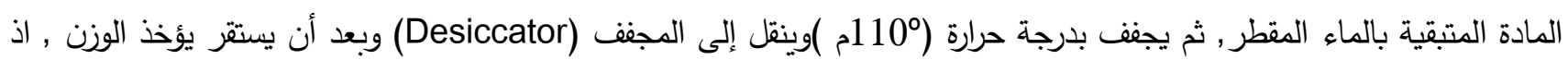
يمثل النقصان بالوزن كمية السيليكا القابلة للتحويل الى سيليكات الصوديوم(11). ان الراشح الناتج من كل خام يمثل محلول سيليكات الصوديوم والذي سيستخدم في تحضير الزيولايت قيد البحث, ان الراسب المتبقي من خام البوكسايت سوف يستخدم في تحضير محلول الومينات الصوديوم • ومن الجدير بالذكر فان هذه الخطوة تسبقها عملية ازالة للكاربونات والجبسوم بالنسبة لخام السيليكا وازالة للكاربونات من فن فئن خام البوكسايت من خلال التصعيد الحراري مع حامض الهيدروكلوريك ذي تركيز (10\%)(12و13). اما الحديد فقد تم ازالته من خلاد تفاعل الخام مع مادة ثنائي ثايونايت الصوديوم ذات تركيز (4\%) لكلا الخامين ايضاً (14).

3-2 يضاف الى المتبقي من خام البوكسايت بعد فصل السيليكا القابلة للتحويل منه , (100) مل من الماء المقطر ثم يوضع على هيتر كهربائي ذو محرك مغناطيسي ويسخن بانتظام عند درجة حرارة (70 - 80)ْ م, ثم يضاف (100) مل من حامض الكبريتيك

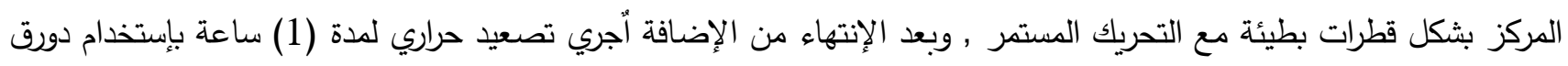
دائري ذي سعة (500) مل, بعدها يتم تبريد المحلول إلى درجة حرارة المختبر ثم يتم ترشيحه وبعدها يغسل الراسب المبت المتبقي بالماء

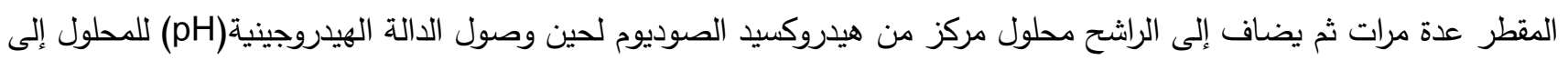
(11) ويحتفظ بالراشح الذ يمثل محلول الومينات الصوديوم الذي سيستخدم لتحضير الزيولايت .

3-3 تحضير الزيولايت

بعد الحصول على المحاليل الاساسية للتحضير (سيليكات والومينات الصوديوم) يتم تفاعل المحلولين , ويضاف (20 مل) من العامل الموجه للبنية ثلاثي أثيل أمين (Tri Ethyl Amine) الى محلول الالومينات بعد ان وضع في بيكر سعة ( 1 لتر ) على الكي الهيتر الكهربائي ذو المحرك المغناطيسي وضبط درجة الحرارة عند (70-80 م) ثم يضاف اليه من الاعلى بواسطة قمع فصل

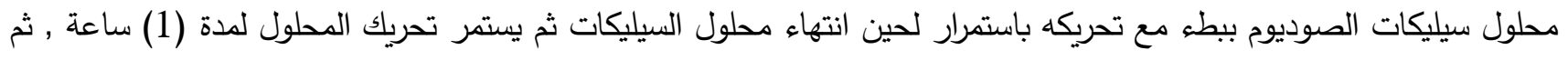
يرفع ويوضع في عبوة محكمة من التفلون لمدة يومين بدرجة حرارة (80) م في داخل الفرن لاكتمال عملية تبلور الزيولايت , بعدها يتم رفع العبوة وتبرد الى درجة حرارة المختبر , ثم يرشح ويغسل الراسب بالماء المقطر عدة مرات لحين التاكد من إنتهاء القاعدة

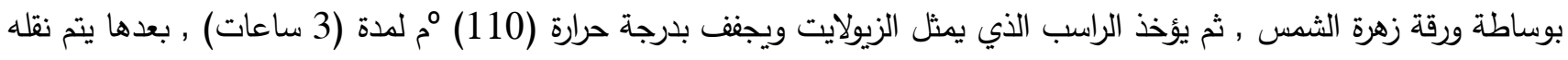

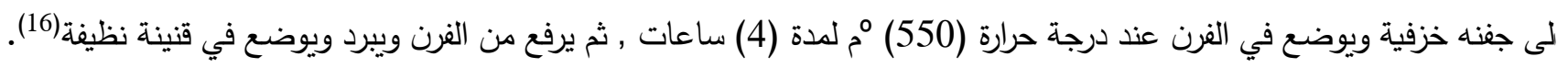

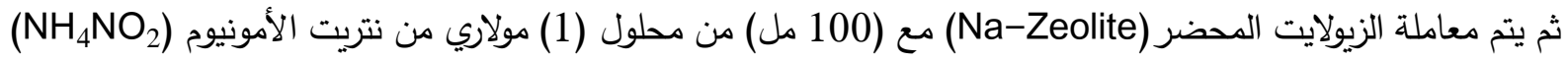
اذ يتم وضعة في دورق دائري سعة (250 مل) ويصعد حرارياً لمدة (2) ساعة عند درجة حرارة (80) مُ م ر ثم ييرد المحلول بدرجة 
حرارة المختبر ويرشح , بعدها يجفف عند درجة حرارية (110) oم لمدة (2) ساعة , اذ إن الراسب المتكون يمثل الزيولايت بصيغة (H-Zeolite)

4-قياس خصائص ومواصفات الزيولايت المحضر : تم اجراء عدد من القياسات على الزيولايت المحضر للتعرف خصائصه ومواصفاته وهي التحليل الحراري الوزني الذي اجري يدويا

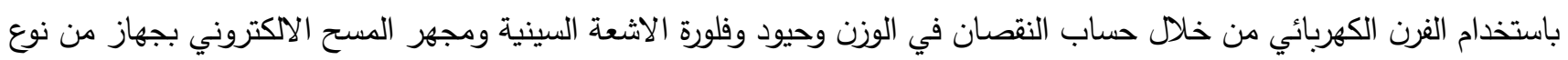
(Fesem Tescan Mira3 France) وتقنية الامتزاز بطريقة(BET) باستخدام جهاز من نوع (Tri Star II Plus).

\section{النتائَج والمناقشة 1 - تحليل الخامات الطينية قيد البحث}

بعد اجراء تحليل خام السيليكا كيميائيا تم الحصول على النتائج الددرجة في الجدول (1)

\begin{tabular}{|c|c|}
\hline Metal Oxides & $\%$ Ratio \\
\hline $\mathrm{SiO}_{2}($ Unreacted $)$ & 8.5 \\
\hline $\mathrm{SiO}_{2}$ (Converted to Silicate) & 31.2 \\
\hline $\mathrm{CaO}$ & 20.55 \\
\hline $\mathrm{SO}_{3}$ & 15.10 \\
\hline $\mathrm{Al}_{2} \mathrm{O}_{3}$ & 3.14 \\
\hline $\mathrm{Fe}_{2} \mathrm{O}_{3}$ & 3.53 \\
\hline MgO & 2.29 \\
\hline Loss On Ignition & 15.48 \\
\hline
\end{tabular}

تبين من الجدول (1) ان هذا الخام يتكون بصورة رئيسية من السيليكا القابلة للتحويل الى سيليكات الصوديوم وهي المادة

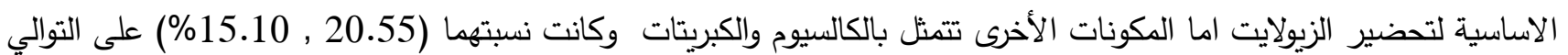
مع احتوائه على تراكيز قليلة من الحديد والمغنسيوم والالمنيوم , في حين كانت نسبة الفقدان بالحرق (15.18 \%) (18).

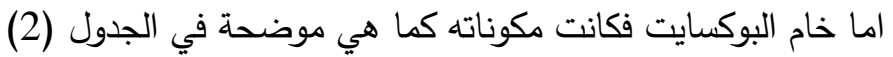

\begin{tabular}{|c|c|}
\hline الأكاسيد العنصرية & النسبة المئوية \% \\
\hline $\mathrm{SiO}_{2}($ Unreacted) & 15.11 \\
\hline $\mathrm{SiO}_{2}($ Converted to Silicate $)$ & 12.2 \\
\hline $\mathrm{Al}_{2} \mathrm{O}_{3}$ & 54.02 \\
\hline $\mathrm{Fe}_{2} \mathrm{O}_{3}$ & 1.34 \\
\hline $\mathrm{CaO}$ & 0.75 \\
\hline MgO & 0.51 \\
\hline $\mathrm{Na} 2 \mathrm{O}$ & 0.41 \\
\hline $\mathrm{K}_{2} \mathrm{O}$ & 0.63 \\
\hline Loss On Ignition & 15.05 \\
\hline
\end{tabular}

يثير الجدول (2) احتواء خام البوكسايت على نسبة عالية من الالمنيوم قدرت ب (54.02 \% ) وهي نسبة عالية وقد تم

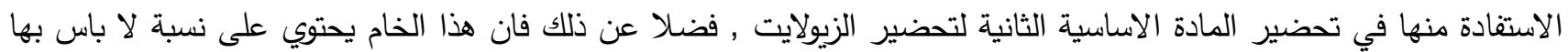


من السيليكا القابلة للتحويل الى سيليكات الصوديوم كانت (12.2\%) مع نسب قليلة من بقية العناصر , بينما كانت نسبة الفقدان بالحرق (15.05\%)(10). كذلك تم قياس حيود الاشعة السينية (XRD) لكل من خام السيليكا والبوكسايت حيث يبين الجدول (3) قيم المسافات الذرية والزوايا(20) للدعادن الموجودة في خام السيليكا. الجدول (3) قيم المسافات الذرية (d-Spacing ) والزوايا (20) للمعادن الموجودة في خام السيليكا.

\begin{tabular}{|c|c|c|c|}
\hline Minerals & Chemical Stracture & $2 \theta$ & $\mathbf{d}\left(\mathbf{A}^{\mathbf{o}}\right)$ \\
\hline Illite & $\mathrm{K}_{2} \mathrm{Al}_{4}\left[\mathrm{Si}_{6} \mathrm{Al}_{2} \mathrm{O}_{29}\right] \mathrm{OH}_{4}$ & $\begin{array}{c}21.95 \\
31.5 \\
33.5 \\
43.8 \\
55.8 \\
57.0 \\
60.50 \\
64.0 \\
74.2\end{array}$ & $\begin{array}{l}4.04 \\
2.84 \\
2.67 \\
2.06 \\
1.65 \\
1.61 \\
1.54 \\
1.45 \\
1.28\end{array}$ \\
\hline Chlorite & $\begin{array}{c}(\mathrm{x}, \mathrm{y})_{4-6}(\mathrm{Si}, \mathrm{Al})_{4} \mathrm{O}_{10}(\mathrm{OH}, \mathrm{O})_{8} \\
\mathrm{X} \text { and } \mathrm{y}=\mathrm{Fe}, \mathrm{Mg}, \mathrm{Mn}\end{array}$ & $\begin{array}{c}24.4 \\
33.9 \\
37.0 \\
39.8 \\
42.9 \\
46.0 \\
76.7 \\
79.95 \\
\end{array}$ & $\begin{array}{c}3.64 \\
2.64 \\
2.43 \\
2.26 \\
2.11 \\
1.97 \\
1.24 \\
1.2 \\
\end{array}$ \\
\hline Gypsum & $\mathrm{CaSO}_{4} \cdot 2 \mathrm{H}_{2} \mathrm{O}$ & $\begin{array}{l}11.8 \\
21.0 \\
29.1 \\
29.3 \\
51.5\end{array}$ & $\begin{array}{l}7.49 \\
4.23 \\
3.06 \\
3.04 \\
1.77\end{array}$ \\
\hline Quartz & $\mathrm{SiO}_{2}$ & $\begin{array}{l}21.3 \\
26.8 \\
36.2 \\
50.3 \\
55.2 \\
66.9 \\
68.4 \\
\end{array}$ & $\begin{array}{c}4.17 \\
3.32 \\
2.48 \\
1.81 \\
1.66 \\
1.4 \\
1.37 \\
\end{array}$ \\
\hline Calcite & $\mathrm{CaCO}_{3}$ & $\begin{array}{l}29.3 \\
48.2 \\
58.8\end{array}$ & $\begin{array}{l}3.04 \\
1.89 \\
1.57\end{array}$ \\
\hline Hematite & $\mathrm{Fe}_{2} \mathrm{O}_{3}$ & $\begin{array}{c}34.95 \\
40.8 \\
41.0 \\
69.2\end{array}$ & $\begin{array}{l}2.56 \\
2.21 \\
2.20 \\
1.36\end{array}$ \\
\hline
\end{tabular}

وتم حساب النسب المئوية للمعادن الطينية وغير الطينية المتواجدة في الخام وكما هو مبين في الجدول ادناه: 
الجدول (4) نسب المعادن الطينية وغير الطينية لخام السيليكا من خلال حيود الأثعة السينية (XRD) .

\begin{tabular}{|c|c|}
\hline Minerals & Percentage Ratio \% \\
\hline Gypsum & 27.081 \\
\hline Quartz & 20.719 \\
\hline Illite & 20.15 \\
\hline Calcite & 20.10 \\
\hline Chlorite & 10.25 \\
\hline Hematite & 1.7 \\
\hline
\end{tabular}

يتبين من الجدول (4) احتواء هذا الخام على المعادن المحتوية على السيليكون في تركيبها بنسبة عالية وهي الايلايت

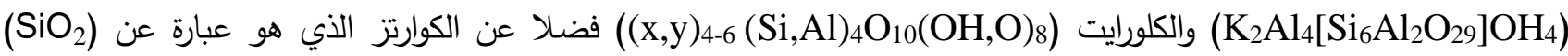
والتي بلغت نسبها مجموعةً (51.119 \% ) وهو يتطابق مع نتائج التحليل الكيميائي الذي اثبت احتواؤه على نسبة عالية من السيليكا • ويوضح الثكل (1) حيود الاشعة السينية لخام السيليكا(19).

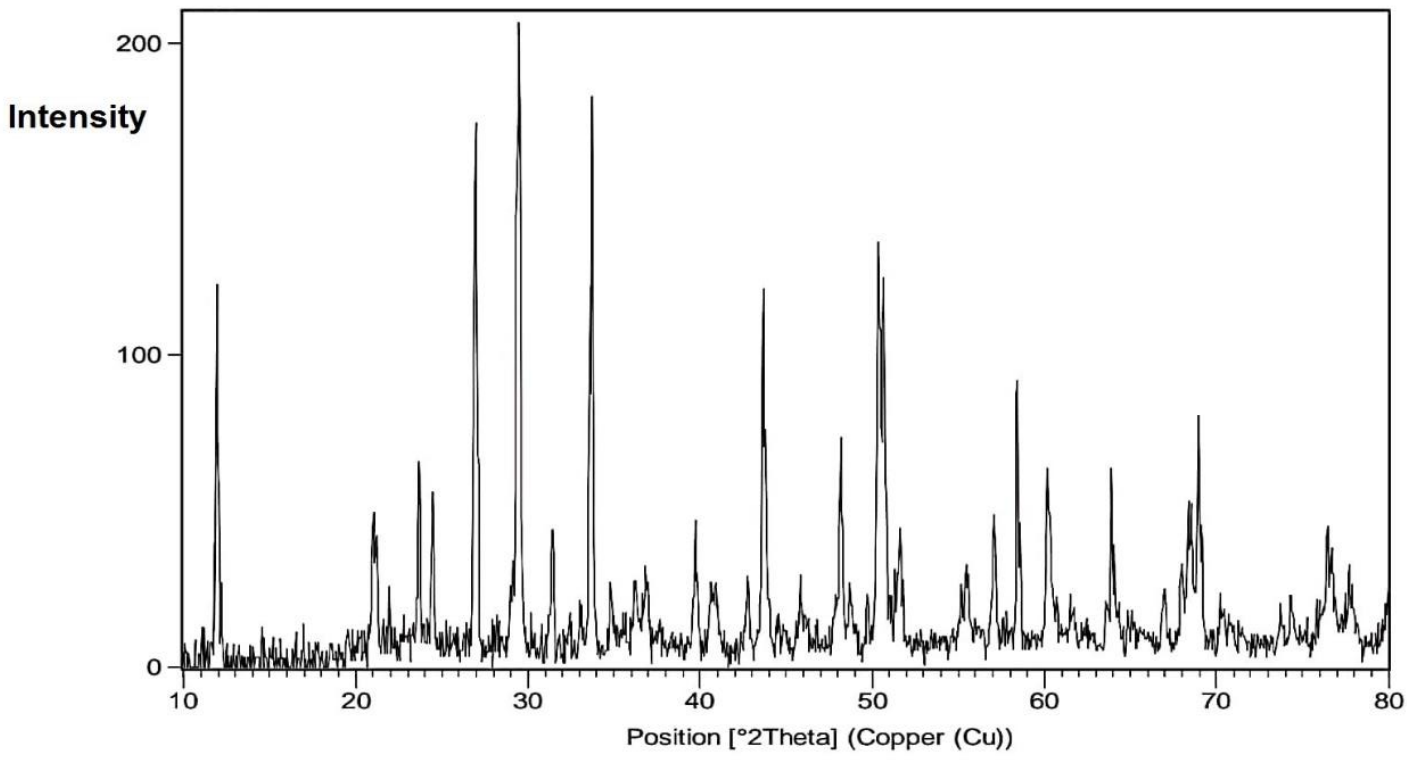

الثكل (1) حيود الاشعة السينية لخام السيليكا.

اما البوكسايت فكانت نسبة المعادن المكونة له كما مبين في الجدول (5) الجدول (5) النسب المئوية للمعادن الطينية وغير الطينية لذام البوكسايت من خلال حيود الأثشعة السينية (XRD) .

\begin{tabular}{|c|c|}
\hline Minerals & Percentage (\%) \\
\hline Boehmite & $\mathbf{3 6 . 1 6 6}$ \\
\hline Kaolinite & $\mathbf{2 0 . 8 1 9}$ \\
\hline Quartz & $\mathbf{1 4 . 5 1 1}$ \\
\hline Halloysite & 12.055 \\
\hline Gibbsite & 10.969 \\
\hline Calcite & $\mathbf{5 . 4 8 0}$ \\
\hline
\end{tabular}




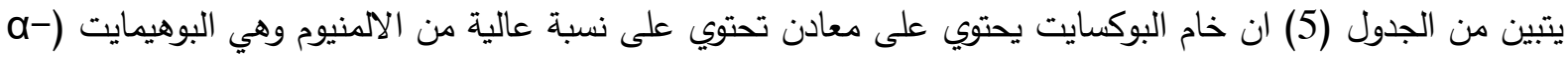

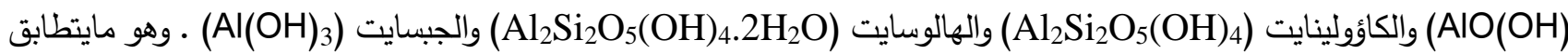
مع نتائج التحاليل الكيميائية للعناصر • وان هذه النسبة يتم تحويلها الى الومينات الصوديوم لغرض تفاعلها مع سيليكات الصوديوم المحضرة سابقا لغرض تحضير الزيولايت(20.ويوضح الثكل(2) حيود الاشعة السينية لخام البوكسايت

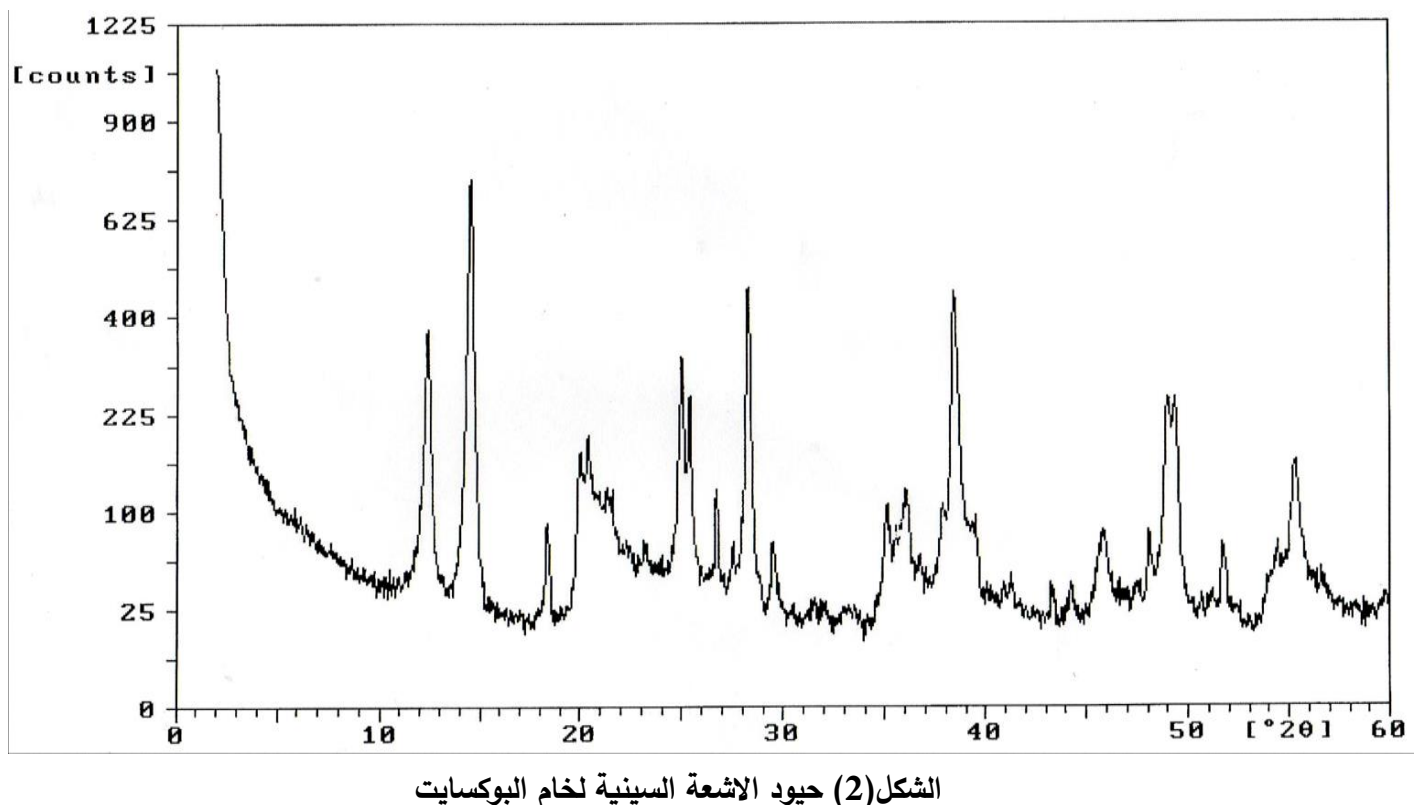

اما نتائج قياس فلورة الاشعة السينية للخامين فكانت كما مبينة في الجدول (6) الجدول (6) نتائج فلورة الاشعة السينية لذامي السيليكا والبوكسايت ومقارنتها مع التحليل الكيميائي

\begin{tabular}{|c|c|c|c|c|}
\hline \multirow[b]{2}{*}{ Metal Oxides } & \multicolumn{2}{|c|}{ Silica Ore } & \multicolumn{2}{|c|}{ Bauxite Ore } \\
\hline & $\begin{array}{c}\text { XRF Results } \\
\text { Wt. \% }\end{array}$ & $\begin{array}{c}\text { Chemical Analysis } \\
\text { Wt.\% }\end{array}$ & $\begin{array}{l}\text { XRF Results } \\
\text { Wt. \% }\end{array}$ & $\begin{array}{c}\text { Chemical Analysis } \\
\text { Wt. \% }\end{array}$ \\
\hline $\mathrm{SiO}_{2}$ & 38.9 & 39.7 & 35.8 & 27.31 \\
\hline $\mathrm{CaO}$ & 22.2 & 20.55 & 0.92 & 0.75 \\
\hline $\mathrm{SO}_{4}^{-2}$ & 15.8 & 15.1 & $-\cdots$ & --- \\
\hline $\mathrm{Al}_{2} \mathrm{O}_{3}$ & 5.4 & 5.07 & 55.5 & 54.02 \\
\hline $\mathrm{Fe}_{2} \mathrm{O}_{3}$ & 1.8 & 1.6 & 1.1 & 1.34 \\
\hline MgO & $-\cdots$ & 2.29 & 0.57 & 0.51 \\
\hline $\mathrm{K}_{2} \mathrm{O}$ & 0.67 & $-\cdots$ & --- & --- \\
\hline $\mathrm{TiO}_{2}$ & 0.382 & --- & $-\cdots$ & -- \\
\hline L.O.I & --- & 15.18 & & 16.5 \\
\hline
\end{tabular}

تبين نتائج فلورة الاشعة السينية (XRF) (20) تقاربهما في اغلب القياسات مع نتائج التحليل الكيميائي لكلا الخامين قيد البحث. بعد التعرف على مكونات الخامات قيد الدراسة والحصول على المواد (سيليكات والومينات الصوديوم) اللازمة لتحضير الزيولايت تم ضبط الدالة الهيدروجينية (pH)عند (11) لانها تعد دالة مثلى لتحضير الزيولايت اما الفائدة من اضافة العامل الموجه للبنية (ثلاثي اثيل امين) هو اسهامه في تحديد حجم المسامات حيث سيتداخل بين الوحدات المتفاعلة للسيليكون والالمنيوم اثثاء عملية التحضير , اما المواد التي تم ازالتها اولا وهي الكاربونات والجبسوم والحديد فهي ذات تاثير سلبي على عملية التحضير لهذا السبب تمت ازالتها قبل عملية التحضير , ان الغرض من تسخين الزيولايت الى درجة حرارة (550م) هو لتنكك العامل الموجه للبنية وازالته من داخل 
المسامات بعد انتهاء دوره وبالتالي زيادة المساحة السطحية الداخلية للزيولايت(21) , اما معاملة الزيولايت بمادة نتريت الامونيوم فكان من اجل تحويل الزيولايت الى الصيغة ذات الفعالية الاكبر من خلال استبدال ايونات الصوديوم بايونات الهيدروجين وبالتالي زيادة المواقع الحامضية الحفازية للزيولايت (17).

$$
\text { 2- القياسات التي اجريت على الزيولايت المحضر }
$$

تم اجراء عدد من القياسات مثلما ذكر في الجزء العملي لغرض التعرف على خصائص ومواصفات الزيولايت المحضر , فقد تم قياس التحليل الحراري الوزني الذي اجري باستخدام الفرن الكهربائي يدويا وتبين ان له اربع عمليات نقصان في الوزن

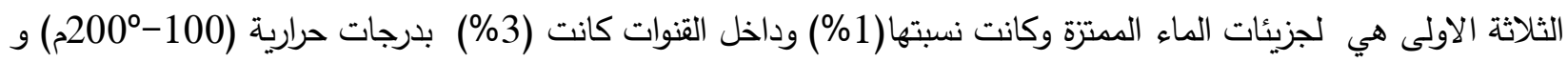
(200-300 م) على التوالي اما الفقدان بين (300-400م) فكان لمجاميع الهيدروكسيل المغادرة على هيئة جزيئات ماء وكانت نسبتها (1\%) أما كمية الفقدان بين (400-550 م م ) تقريباً فتعزى إلى فقدان جزيئات العامل الموجه للبنية, ويلاحظ

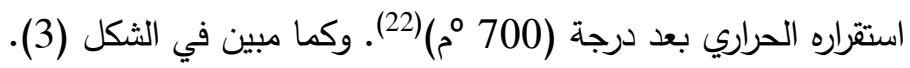

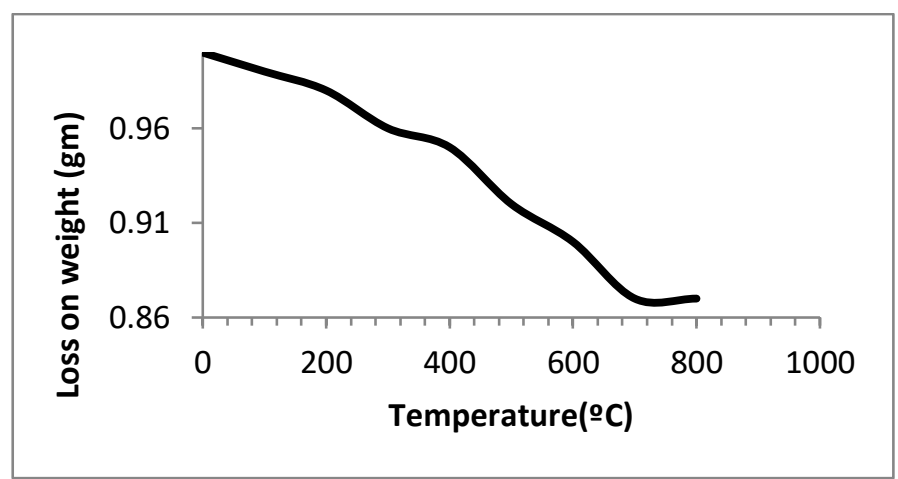

الثكل (3) التحليل الحراري الوزني للزيولايت المحضر

اما نتائج حيود الاشعة السينية (XRD) فقد تبين من خلال نموذج الحيود كما مبين في الثكل (4) أن الزيولايت المحضر يمتلك نظاماً بلورياً مختلفاً عن الأنماط المعروفة إذ أن الإمتصاص الرئيسي يظهر في موقع (20) قيمته (67) وكما مبين في الثكل

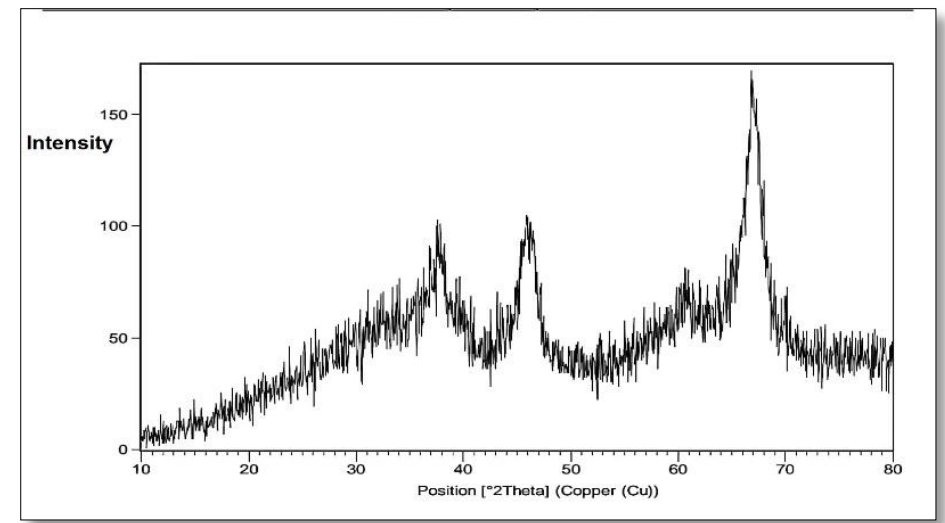

لثكل (4) حيود الاشعة السينية للزيولايت المحضر

ويمكن توضيح قيم معاملات ميلر والزوايا (20) والمسافات الذرية للزيولايت المحضر كما في الجدول (7): 
الجدول (7) قيم معاملات ميلر والزوايا(20) والمسافات الذرية(d-spacing) للزيولايت المحضر من خلال قياس(XRD)

\begin{tabular}{|c|c|c|}
\hline Miller Indices & $\mathbf{2 \theta}$ & $\mathbf{d}$-spacing( $\mathbf{A}^{\mathbf{0}} \mathbf{)}$ \\
\hline \hline 220 & 30.8 & 2.90 \\
\hline 400 & 31.8 & 2.81 \\
\hline 210 & 38 & 2.36 \\
\hline 440 & 46 & 1.97 \\
\hline 111 & 60.5 & 1.53 \\
\hline 533 & 67 & 1.39 \\
\hline 200 & 70 & 1.34 \\
\hline
\end{tabular}

وإذا ما شاهدنا قيم معاملات ميلر في الجدول (7) والتي كانت (220) , (400) , (210) , (440) , (111) , (333)

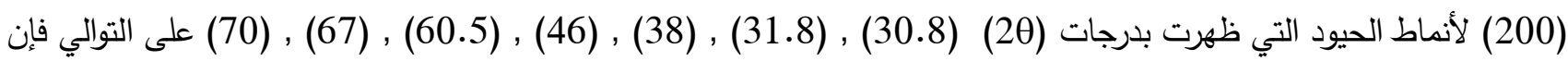
النظام البلوري أقرب ما يكون إلى النظام المكعبي , إن ظهور أنماط الحيود للزيولايت إنما يدل على الطريقة الصحيحة التي أتبعت في التحضير والتي أنتجت زيولايت ذا نظام بلوري ممتاز وكذلك يدل على نسبة التبلور الجيدة من خلال ملاحظة شدة أنماط الحيود (19).

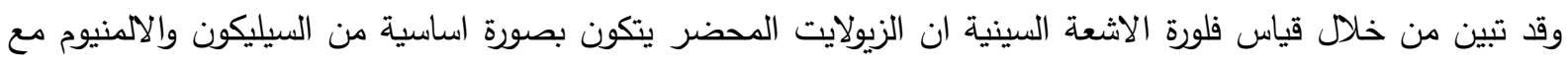

كميات قليلة من باقي العناصر (20) وكما مبين في فلرف الجدول (8)

الجدول (8) النسب المئوية للعناصر المتواجدة في الزيولايت المحضر من خلال فلورة الأثعة السينية (XRF) .

\begin{tabular}{|c|c|}
\hline Elemental Oxides & Wt. (\%) \\
\hline $\mathrm{SiO}_{2}$ & 50.05 \\
\hline $\mathrm{Al}_{2} \mathrm{O}_{3}$ & 40.13 \\
\hline $\mathrm{TiO}_{2}$ & 3.6 \\
\hline $\mathrm{CaO}$ & 2.72 \\
\hline $\mathrm{MgO}$ & 1.7 \\
\hline $\mathrm{K}_{2} \mathrm{O}$ & 0.732 \\
\hline $\mathrm{Fe}_{2} \mathrm{O}_{3}$ & 0.72 \\
\hline $\mathrm{Na}_{2} \mathrm{O}$ & 0.302 \\
\hline
\end{tabular}

واظهرت نتائج قياس المجهر الالكتروني الماسح أن للزيولايت المحضر تجمعات كروية متباينة الحجم ومشوهة قليلاً وقسم من هذه الحبيبات قد تجمع لتشكل حبيبات أكبر حجماً, وكذلك أظهر هذا القياس النظام المسامي العالي للزيولايت الذي له دور فاعل في عملية تحفيز التفاعلات ـ أما ما يخص حجم الحبيبات فكانت بحدود (18.17nm) بعضها أكبر من ذلك فكانت (23.96nm) و (32.56nm) , إن حجم الحبيبات الذي تم ملاحظته بعدد قليل من النانو مترات يدل على النوعية الممتازة لهذا الزيولايت وفعاليته

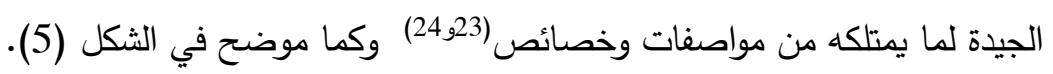




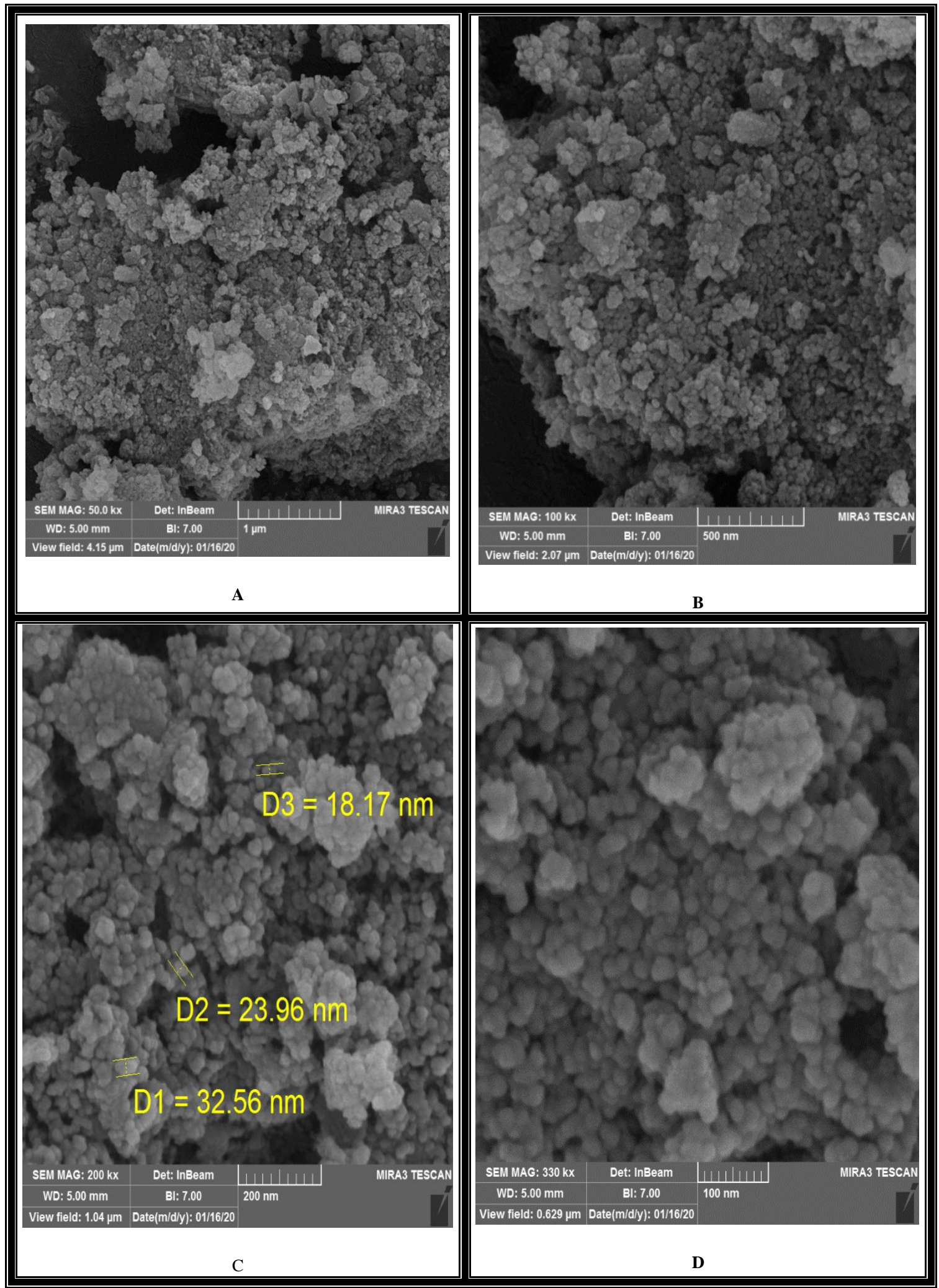

الثكل (5) : صور المجهر الاكتروني الماسح بتكبيرات مختلفة للزيولايت المحضر . $(100 \mathrm{~nm}=\mathrm{D}, 200 \mathrm{~nm}=\mathrm{C}, 500 \mathrm{~nm}=\mathrm{B}, 1 \mu \mathrm{m}=\mathrm{A})$

وقد بينت نتائج قياس الزيولايت بتقنية (BET) انه ذو مواصفات جيدة من حيث المساحة السطحية وقطر وحجم المسامات

وكما موضح في الجدول (9). 
الجدول (9) قيم المساحة السطحية وحجم وقطر المسامات للزيولايت المحضر من خلال قياس (BET) .

\begin{tabular}{|c|c|}
\hline Measurements & Analysis Data \\
\hline Surface Area & $244.3766 \mathrm{~m}^{2} / \mathrm{g}$ \\
\hline Pore Volume & $0.591209 \mathrm{~cm}^{3} / \mathrm{g}$ \\
\hline Pore Size & $9.9384 \mathrm{~nm}$ \\
\hline
\end{tabular}

يلاحظ من الجدول (9) المساحة السطحية العالية للزيولايت المحضر فقد كانت (244.3766 م² غم) , اما حجم المسامات الذي يمثل الفراغ بين حبيبات الزيولايت فكان (0.591209 سم غم) وهو يدل على صغر ودقة حجم الحبيبات , اما قطر المسامات

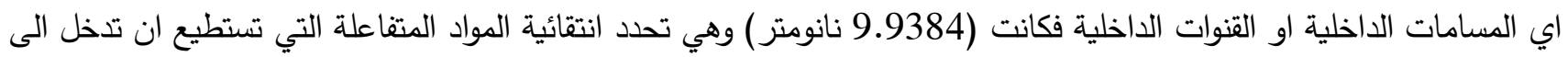
داخل قنوات الزيولايت اعتمادا على حجمها الجزيئي (26.25).

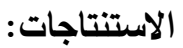
ان الزيولايت المحضر ذو مواصفات جيدة مثل المساحة السطحية وامتلاكه درجة تبلور وقدرة امتزازية عاليتين يمكن من خلالها استخدامه كحفاز وهذا ياتي بالاستفادة من المحتوى الجيد للخامات المستخدمة في الحصول على المواد الاولية لتحضير الزيولايت وكذلك بفضل استخدام العامل الموجه للبنية (ثلاثي مثيل امين) , علما انه قد تم تطبيق الحفاز في دراسة ستتشر لاحقا وتبين قدرته التحفيزية العالية في مجال الاصلاح التركيبي للنفثا.

1. Auerbach, S.M.,Carrado, K.A. and Dutta, P.K. (2003). "Handbook of Zeolite Science and Technology", Marcel Dekker, Inc., p.p.1-8.

2. Niwa, M., Katada, N., Okumura, K. (2010). "Characterization and Design of Zeolite Catalyst", Springer-Verlag. Germany, p.p.7-20.

3. Murrgy, H.H. (2007). "Applied Clay Mineralogy", Elsevier B.V., 1st Ed., p.p.111-130.

4. Kulprathipanja, S. (2010). "Zeolite in Industrial Separation and Catalyst", Wiley VCH Verlag, p.528.

5. Al-Hemiri, A.A. and Jawad, Y., (2009) ," Differences Between Sodium Metasilicateand Silicic Acid as Silica Source for Zeolite Y Nano particles Synthesis by Sol-Gel Method" Iraqi Journal of Chemical and Petroleum Engineering IJCPE, Vol. (10), No. (4), p.p.13-17.

6. Rahman , M.M. , Awang, M.B. and Yusof , A.M., (2012), "Preparation, characterization and application of zeolite-Y (Na-Y) for water filtration" , Australian Journal of Basic and Applied Sciences, Vol. (6), No. (1), p.p.50-54.

7. Barot, S. , Maheria , K.C. and Bandyopadhyay, R. , (2016), "Synthesis of SAPO-34 molecular sieves by varying synthetic parameters and study of its effect on Biginelli reaction" , J. Mater Environ Science JMES, Vol. (7), No.(3), p.p. 899-906. 
8. Mohiuddin , E. , Mdleleni , M.M. and Key, D., (2018), Applied Petrochemical Research, Vol. (8), No. (2), p.p.119-129.

9. Jeffery, P.G. and Hutchison, D. (1981), "Chemical Methods of Rock Analysis", $3^{\text {rd }}$ Ed., Pergamon Press, p.p.30-39.

10. Ghazal , R.Y. , (2011), "Preparation of Some Alkylation Catalyst From Natural Clays and Their Uses in the Production of Linear Alkyl Benzene (LAB)" , Ph. D. Thesis University of Mosul, (In Arabic).

11. Nibori , Y. , Kunita , M. , Tochiyama , O. and Chida , T. , (2000), "Dissolution Rates of Amorphous Silica in Highly Alkaline Solution" , Journal of Nuclear Science and Technology , Vol. (37), No. (4), p.p. 349-357.

12. Adekola , F.A., Olosho, A.I. , Baba , A.A. and Adebayo, S.A., (2018), "Dissolution Kinetics Studies of Nigerian Gypsum Ore in Hydrochloric Acid" , Journal of Chemical Technology and Metallurgy, Vol.( 53), No.( 5), p.p. 845-855.

13. Loeppert, R.H. and Suarez, D.L., (1996), "Carbonate and Gypsum", Publication From USDA - ARS / UNL / Faculty, University of Nebraska , Lincoln .

14. Gougazeh , M. , (2018), "Removal of Iron and Titanium Contaminants From Jordanian Kaolin's By Using Chemical Leaching" , Journal of Taibah University of Science , Vol. (10) , p.p. $1-8$.

15. China , C.R. , (2019), "Preparation of Aluminum Sulphate From Kaolin and its Performance in Combination Tanning" , SN Applied Science Journal, Vol. (1), Research Article .

16. Martinez , J.G. and Kunhao, L. , (2015), "Mesoporous Zeolites, Preparation, Characterization and Applications", Wiley - VCH Verlag GmbH \& Co. KGaA ,p.p. 11- 21.

17. Abdul Halim , A.K. , Camer, S.K. and Rahman , Am. , (2010), "Characterization and Cracking Activity of Zeolite Prepared Form Local Kaolin", Iraqi Journal of Chemical and Petroleum Engineering, Vol. (11), No.(2), p.p. 35-42 .

18. Estefan , G. , Sommer, R. and Ryan, J. , (2013), "Methods of Soil , Plant and Water Analysis , $3^{\text {rd }}$. Ed. , ICARDA Publishing, p.p. $61-68-80-108-111-120$.

19. Che , M. and Redrine , J. ,(2012),"Characterization of Solid Materials and Heterogeneous Catalysts", Wiley-VCH Verlag \& Co. KGaA. p.p.611-640 .

20. Leng ,Y. , (2013), "Materials Characterization" ,Wiley -VCH Verlag \& Co. KGaA.,p.p.4762,196 .

21. Jong , K. P. ,(2009) ,"Synthesis of Solid Catalyst" , Wiley -VCH Verlag \& Co. KGaA , Weinhiem, p.p. 83-98. 
22. Brow , M.E. , (2004) , "Introduction to Thermal Analysis", 2nd . Ed." , Kluwer Academic Publisher, p.p. 19-28 .

23. Rao , C.N.R. and Biswas , K. , (2009) , "Characterization of Nanomaterials by Physical Methods" , Annual Review of Analytical Chemistry Vo 1. (2) , No.(1) , p.p. 435-462 .

24. Naito , M. , Yokoyama , T. , Hosokawa , K. and Nogi , K. , (2018), "Nanoparticle Technology Handbook", 3rd . Ed , Elsevier, p.p.23-25 .

25. Toth , J. , (2001) , "Adsorption ; Theory , Modeling and Analysis" , Marcel Dekker , Inc ., p.p. $68-73,80-81$.

26. Tien , C. , (2019) , "Introduction to Adsorption , Basics , Analysis and Application" , Elsevier Inc. , p.p. 31-39 